\title{
ITGB5 and AGFG1 variants are associated with severity of airway responsiveness
}

\author{
Blanca E Himes ${ }^{1,2,3^{*}}$, Weiliang Qiu', Barbara Klanderman², John Ziniti ${ }^{1}$, Jody Senter-Sylvia', Stanley J Szefler ${ }^{4}$, \\ Robert F Lemanske, $\mathrm{Jr}^{5}$, Robert S Zeiger ${ }^{6}$, Robert C Strunk', Fernando D Martinez ${ }^{8}$, Homer Boushey ${ }^{9}$, \\ Vernon M Chinchilli ${ }^{10}$, Elliot Israel ${ }^{11}$, David Mauger ${ }^{10}$, Gerard H Koppelman ${ }^{12}$, Maartje AE Nieuwenhuis ${ }^{13}$, \\ Dirkje S Postma ${ }^{13}$, Judith M Vonk ${ }^{14}$, Nicholas Rafaels ${ }^{15}$, Nadia N Hansel ${ }^{15}$, Kathleen Barnes ${ }^{15}$, Benjamin Raby ${ }^{1}$, \\ Kelan G Tantisira' ${ }^{1}$ and Scott T Weiss ${ }^{1,2}$
}

\begin{abstract}
Background: Airway hyperresponsiveness (AHR), a primary characteristic of asthma, involves increased airway smooth muscle contractility in response to certain exposures. We sought to determine whether common genetic variants were associated with AHR severity.

Methods: A genome-wide association study (GWAS) of AHR, quantified as the natural log of the dosage of methacholine causing a 20\% drop in $\mathrm{FEV}_{1}$, was performed with 994 non-Hispanic white asthmatic subjects from three drug clinical trials: CAMP, CARE, and ACRN. Genotyping was performed on Affymetrix 6.0 arrays, and imputed data based on HapMap Phase 2, was used to measure the association of SNPs with AHR using a linear regression model. Replication of primary findings was attempted in 650 white subjects from DAG, and 3,354 white subjects from LHS. Evidence that the top SNPs were eQTL of their respective genes was sought using expression data available for 419 white CAMP subjects.
\end{abstract}

Results: The top primary GWAS associations were in rs848788 (P-value 7.2E-07) and rs6731443 (P-value 2.5E-06), located within the ITGB5 and AGFG1 genes, respectively. The AGFG1 result replicated at a nominally significant level in one independent population (LHS P-value 0.012), and the SNP had a nominally significant unadjusted P-value (0.0067) for being an eQTL of AGFG1.

Conclusions: Based on current knowledge of ITGB5 and AGFG1, our results suggest that variants within these genes may be involved in modulating AHR. Future functional studies are required to confirm that our associations represent true biologically significant findings.

Keywords: Asthma, Airway hyperresponsiveness, Genome-wide association study, ITGB5, AGFG1

\section{Background}

Asthma is a common chronic respiratory disease affecting over 25 million Americans and its prevalence has risen over the past decade [1]. Airway hyperresponsiveness (AHR), in which the muscles of the airway contract in response to certain exposures, is one of the primary characteristics of asthma and one that has been correlated with

\footnotetext{
* Correspondence: blanca.himes@channing.harvard.edu

'Channing Division of Network Medicine, Brigham and Women's Hospital and Harvard Medical School, Boston, MA, USA

${ }^{2}$ Partners HealthCare Center for Personalized Genetic Medicine and Harvard Medical School, Boston, MA, USA

Full list of author information is available at the end of the article
}

current asthma severity and future lung function [2-4]. Bronchoprovocation challenges with methacholine or histamine have been widely used to quantify AHR in clinical and research settings [5]. These tests consist in administration of increasing dosages of a bronchoconstrictor until a specific decrease in lung function is measured (e.g., 20\% drop in $\mathrm{FEV}_{1}$ ). The mechanisms by which AHR occurs are not fully understood, but AHR has been related directly to changes in airway smooth muscle contractility as well as inflammation and airway remodeling $[6,7]$. Asthma is a disease with demonstrated heritability in humans, and several genes, including the IKZF3ZPBP2-GSDMB-ORMDL3 locus, HLA-DQ, IL1RL1, IL33,

\section{Biomed Central}

(c) 2013 Himes et al.; licensee BioMed Central Ltd. This is an Open Access article distributed under the terms of the Creative Commons Attribution License (http://creativecommons.org/licenses/by/2.0), which permits unrestricted use, distribution, and reproduction in any medium, provided the original work is properly cited. 
TSLP, SLC22A5, SMAD3, and RORA have been consistently associated with asthma in genome-wide association studies (GWAS) [8,9]. Although AHR is often used as a quantifiable and reproducible surrogate of asthma in animal model studies of asthma genetics $[10,11]$, the genetics of AHR have not been widely studied in humans. Nonetheless, the heritability of AHR is supported by a previous twin study [12] and positional cloning and linkage analysis studies of AHR [13,14]. Our goal was to measure the association of genetic variants with AHR severity via a GWAS.

We utilized data from 994 non-Hispanic white asthma patients to measure the association of single nucleotide polymorphisms (SNPs) with severity of AHR and found that the strongest associations were at variants in two genes: ArfGAP with FG repeats 1 (AGFG1) and integrin, beta 5 (ITGB5). After attempting to replicate primary findings in two independent populations (one composed of subjects with asthma and one composed of patients with COPD) and searching for evidence that variants modified expression levels of their respective genes, we found additional evidence to support the involvement of AGFG1 in AHR. Evidence that ITGB5 was involved in AHR was found in previous functional studies of human airway smooth muscle cells.

\section{Methods}

\section{Subjects}

The primary group of subjects consisted of 994 nonHispanic white asthmatics from the Childhood Asthma Management Program (CAMP) [15], and subsets of clinical trials within the Childhood Asthma Research and Education (CARE) network [16], and the Asthma Clinical Research Network (ACRN) [17] participating in the NHLBI SNP Health Association Resource Asthma
Resource project (SHARP). Some demographic characteristics of these cohorts are in Table 1 and further details are provided in the Additional file 1. For each trial, methacholine challenge tests were performed according to American Thoracic Society criteria [18]. Baseline AHR measures were utilized, and AHR was quantified as the natural log of the provocative concentration of methacholine that caused a $20 \%$ decrease in $\mathrm{FEV}_{1}(\mathrm{LnPC} 20)$.

\section{Genotyping and quality control}

Genome-wide SNP genotyping for SHARP subjects was performed by Affymetrix, Inc. (Santa Clara, CA) using the Affymetrix Genome-Wide Human SNP Array 6.0. Dataset quality control (QC) included the removal of related subjects (i.e. CAMP and CARE siblings), subjects with missingness $>5 \%$, SNPs with minor allele frequency (MAF) $<0.05$, missingness $>5 \%$, or not passing HardyWeinberg equilibrium based on a threshold of $1 \mathrm{E}-03$. The genomic inflation factor $\left(\lambda_{\mathrm{GC}}\right)$ for the remaining 442,036 SNPs genotyped in SHARP subjects was 1.007, demonstrating minimal population stratification. Further genotyping and $\mathrm{QC}$ measures are provided in the Additional file 1.

\section{Statistical analysis}

Imputation of all SNPs available in HapMap Phase 2 Release $22 \mathrm{CEU}$ data using the Markov Chain Haplotyping software $(\mathrm{MaCH})$ [19] was performed for SHARP genotype data. The primary GWAS was based on the set of 2,154,322 imputed SNPs with MAF $>0.05$ and a ratio of empirically observed dosage variance to the expected (binomial) dosage variance greater than 0.3 , indicating good quality of imputation. The association of SNPs

Table 1 Characteristics of GWAS subjects

\begin{tabular}{|c|c|c|c|c|c|}
\hline & $\begin{array}{c}\text { CAMP } \\
(n=525)\end{array}$ & $\begin{array}{c}\text { CARE }^{\S} \\
(n=195)\end{array}$ & $\begin{array}{c}\text { ACRN } \\
(n=274)\end{array}$ & $\begin{array}{c}\text { LHS } \\
(n=3,354)\end{array}$ & $\begin{array}{c}\text { DAGl } \\
(n=650)\end{array}$ \\
\hline Age - Mean (SD) & $8.8(2.1)$ & $10.6(2.9)$ & $31.5(10.3)$ & $48.8(6.7)$ & $36.0(13.8)$ \\
\hline Gender - N (\%) Male & $322(61)$ & $123(63)$ & $118(43)$ & $1949(58.1)$ & $291(45)$ \\
\hline Wash-out Prior to Methacholine Test* - Weeks & 4 & $0-4$ & $1-6$ & - & - \\
\hline $\operatorname{LnPC20}$ & $0.096(1.21)$ & $-0.25(1.35)$ & $0.80(0.68)$ & $1.58(0.93)$ & $0.71(1.63)$ \\
\hline Current smokers & $0(0)$ & $0(0)$ & $0(0)$ & 3,354 (100) & $106(16)$ \\
\hline Baseline $\mathrm{FEV}_{1}$ & $1.7(0.5)$ & $2.2(0.8)$ & $3.2(0.8)$ & $2.7(0.6)$ & $2.8(0.9)$ \\
\hline Baseline FEV 1 \% Predicted & $94.4(14.1)$ & $98.8(13.0)$ & $86.8(13.5)$ & $78.2(10.0)$ & $82.3(20.5)$ \\
\hline Height $[\mathrm{cm}]$ & $132.8(13.6)$ & $143.4(16.8)$ & $170.3(10.0)$ & $171.3(8.9)$ & $172.1(11.5)$ \\
\hline Weight [kg] & $32.4(10.9)$ & $42.9(18.1)$ & $75.6(18.2)$ & $75.1(14.8)$ & $74.7(17.6)$ \\
\hline $\mathrm{BMI}\left[\mathrm{kg} / \mathrm{cm}^{\wedge} 2\right]$ & $17.9(3.1)$ & $20.1(4.9)$ & $25.9(5.3)$ & $25.5(3.9)$ & $25.0(4.8)$ \\
\hline
\end{tabular}

*Subjects were allowed to use rescue medication during wash-out period.

${ }^{\S}$ CARE trials included: CLIC, PACT, MARS.

"ACRN trials included: BAGS, DICE, IMPACT, PRICE.

IThe LnPC20 of the DAG cohort was calculated for 600 of the 650 asthmatics in the analysis.

Mean (Standard deviation) reported unless otherwise noted. CAMP, CARE, ACRN, and DAG are composed of subjects with asthma and not COPD, while LHS is composed of subjects with COPD who did not have asthma at the time of enrollment but may have had a past history of asthma. 
with LnPC20 was measured with a linear regression model using dosage data as implemented in PLINK [20]. To be sure that demographic characteristics did not have a strong influence on the association results, an additional association model was created using sex, age, height, and study as covariates. Plots of association results near specific genes were created using LocusZoom with the hg18/HapMap Phase II CEU GenomeBuild/LD Population [21]. Combined P-values with replication populations were computed using Fisher's combined probability method [22] where hypothesis tests in replication populations had one-sided alternatives, based on the direction of the association in SHARP, so that SNPs with association tests in opposite directions would not produce inappropriately small P-values. Replication of SNPs with P-values $<1 \mathrm{E}-05$ in the primary GWAS was attempted in two independent cohorts.

\section{Genome-wide gene expression data}

The Asthma BRIDGE project collected mRNA expression level data from 2,520 mRNA microarrays on 210 Illumina HumanHT-12 chips, among which 218 arrays were genetic control arrays and 2,302 arrays were sample arrays from 1,481 subjects. We focused on the 419 arrays (47,053 gene probes) from whole blood samples of 419 white CAMP subjects utilized in the current study. Expression data were $\log 2$ transformed and quantile normalized. The R Bioconductor GGtools package (version 3.11.27) was used to perform cis-eQTL analysis, in which chi squared tests were used to assess whether genotypes of a SNP within $50 \mathrm{~KB}$ from both ends of a gene were associated with the expression levels of the gene after adjusting for age and gender [23].

\section{Replication study}

Dutch Asthma GWAS (DAG). This cohort was comprised of 650 DAG subjects with doctor-diagnosed asthma and documented AHR [14,24]. All subjects had smoking history and steroid use data available at the time of the AHR test. Participants with known AHR but in remission during the test were excluded. Remission was defined as not on steroids and without $20 \%$ or greater fall in $\mathrm{FEV}_{1}$ during the AHR test. The AHR test was conducted using histamine or methacholine as a stimulus. AHR was quantified as the difference between $\mathrm{FEV}_{1}$ at baseline and at the dose step at which a $20 \%$ or greater $\mathrm{FEV}_{1}$ drop was achieved, divided by the dose of stimulant used (slope). Because two protocols were used, one with a 30-second tidal breathing method and a second with a 2-minute tidal breathing phase, the AHR slopes measured with the 30 -second tidal breathing method were divided by 4 , in order to compensate for the 4 times greater duration of administration of stimulus. Slope values were log-transformed so that they would follow a normal distribution. To compare DAG subjects to those of the primary GWAS, PC20 was calculated in 600 of the 650 subjects that had a $20 \%$ or greater drop in $\mathrm{FEV}_{1}$ at the highest provocation dose. Genotyping was performed using the Hapmap $317 \mathrm{~K}$ platform or Illumina 370 Duo Chip. Tests of AHR association were performed via linear regression, with smoking and inhaled/oral steroid use as covariates using PLINK.

Lung Health Study (LHS). This cohort consisted in a subset of 3,354 individuals with COPD from the multicenter (10 centers) North American LHS [25]. The initial study population consisted of 5,887 men and women (63\% male) who were smokers (aged 35-60) at enrollment with spirometric evidence of mild to moderate lung function impairment. While a history of asthma was not a criterion for study exclusion, subjects were excluded if they regularly used asthma medications, including bronchodilators and glucocorticoids. For the AHR GWAS, the subset of European American LHS participants with adequate DNA and valid methacholine measurements at baseline was utilized. Spirometry was performed following ATS criteria, and details of the methacholine challenge tests have been provided previously [26]. Samples were genotyped using the Illumina Human660W-Quad v.1_A BeadChip. Genotyped SNPs were imputed from CEU using 1000 Genomes Project data as a reference. Association of SNPs with $\log 10$ of PC20 was measured using linear regression under an additive model and while adjusting for gender, age at baseline, clinic site, log10(weight in kg), FEV1 (in L), and FEV1/FVC.

The current study was approved by the Partners $\mathrm{Hu}$ man Research Committee (Partners HealthCare, Inc., Boston, MA). Collection of data for the existing human cohort studies was approved by the Institutional Review Board of the corresponding institution(s), which ensured that all procedures followed were in accordance with the ethical standards of the responsible committee on human experimentation, as detailed in the cited works. Informed consent was obtained for all study participants.

\section{Results}

Characteristics of all subjects are provided in Table 1. The primary GWAS measured the association of SNPs with AHR in 994 non-Hispanic white subjects. The quantile-quantile (QQ) plots for both imputed and genotyped SNPs revealed that the distributions of association P-values were similar to those expected for a null distribution [Figure 1]. The lowest P-values $(<1 \mathrm{E}-05$; Table 2$)$ among the primary GWAS are in four regions, two of which correspond to genes: ArfGAP with FG repeats 1 (AGFG1) and integrin, beta 5 (ITGB5) [Figure 2; Additional file 1: Table S1]. Comparison of primary 


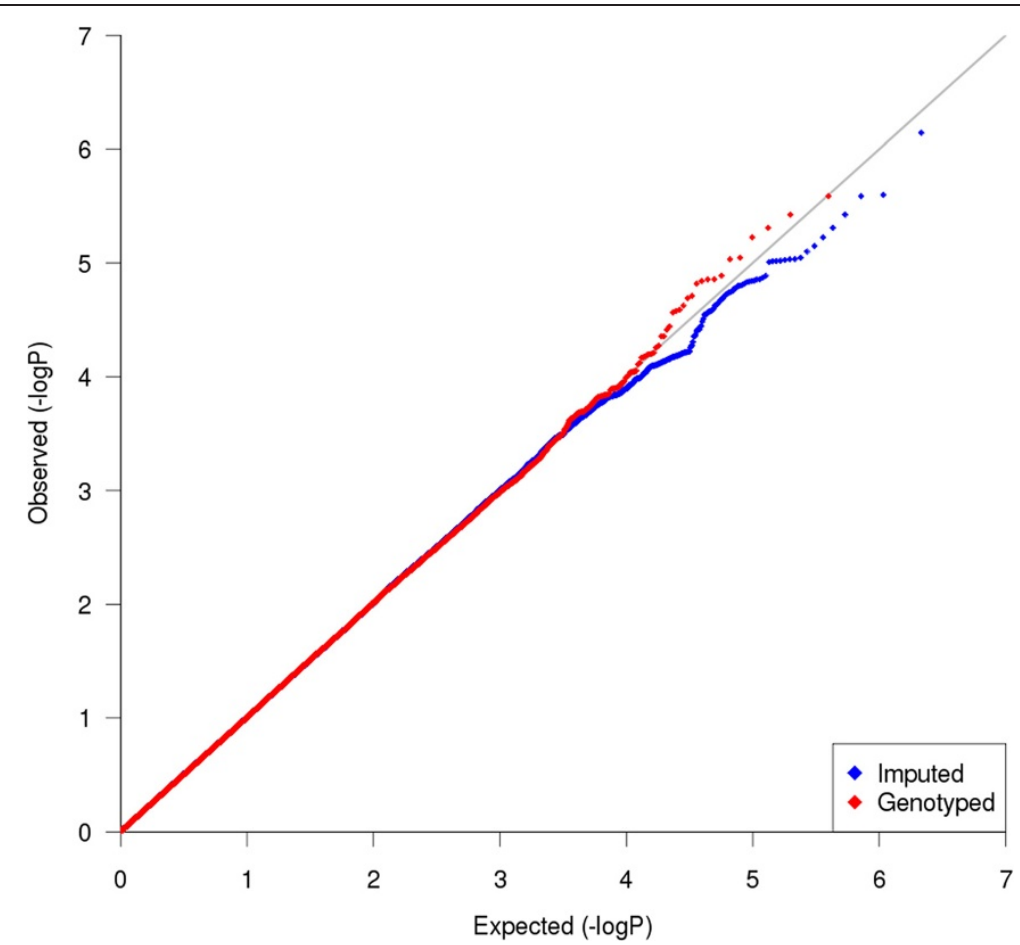

Figure 1 Quantile-quantile (QQ) plot. Comparison of primary GWAS P-values to those expected for a null distribution. Distribution of genotyped (red) and imputed (blue) GWAS results shows little evidence of deviation of measures at the tail, making the distinction among SNPS having low P-values representing true associations vs. those SNPs having low P-values by chance challenging.

association results to those generated with a model that adjusted for sex, age, height, and study revealed that there were no great changes in rankings or P-values, particularly for the top SNPs [Figure 3]. Because some of the top-ranked associations could represent biologically meaningful ones, we proceeded to attempt to replicate them in two independent populations. The SNPs in/near AGFG1 replicated at a nominally significant level (i.e. P-value $<0.05$ ) in LHS, while no replication was obtained in DAG [Table 3].

Table 2 Primary GWAS top results (P-values <1E-05)

\begin{tabular}{|c|c|c|c|c|c|c|c|c|c|}
\hline SNP & CHR & BP & Reference allele & Frequency & Rsq & BETA & SE & P-value & Gene \\
\hline rs848788 & 3 & 126011114 & $C$ & 0.36 & 0.97 & -0.28 & 0.06 & $7.2 \mathrm{E}-07$ & ITGBS \\
\hline rs6731443 & 2 & 228105810 & G & 0.55 & 1.00 & -0.25 & 0.05 & $2.5 \mathrm{E}-06$ & AGFG1 \\
\hline rs13382948 & 2 & 228127201 & A & 0.45 & 1.00 & 0.25 & 0.05 & $2.6 \mathrm{E}-06$ & AGFG1 \\
\hline rs10189795 & 2 & 228087105 & C & 0.45 & 1.00 & 0.25 & 0.05 & $3.8 \mathrm{E}-06$ & AGFG1 \\
\hline rs4861175 & 4 & 41932655 & A & 0.18 & 1.00 & -0.32 & 0.07 & $4.9 \mathrm{E}-06$ & - \\
\hline rs614664 & 3 & 125969683 & A & 0.37 & 1.00 & -0.25 & 0.06 & $6.0 \mathrm{E}-06$ & ITGB5 \\
\hline rs702036 & 3 & 125983762 & A & 0.63 & 0.98 & 0.25 & 0.06 & $7.1 \mathrm{E}-06$ & ITGB5 \\
\hline rs296282 & 5 & 106163683 & C & 0.22 & 0.56 & 0.38 & 0.09 & $8.0 \mathrm{E}-06$ & - \\
\hline rs4972983 & 2 & 228101412 & A & 0.57 & 1.00 & -0.24 & 0.05 & $9.0 \mathrm{E}-06$ & $A G F G 1$ \\
\hline rs7592613 & 2 & 228159434 & C & 0.61 & 1.00 & -0.24 & 0.05 & $9.3 \mathrm{E}-06$ & AGFG1 \\
\hline rs11683662 & 2 & 228156272 & A & 0.61 & 1.00 & -0.24 & 0.05 & $9.3 \mathrm{E}-06$ & AGFG1 \\
\hline rs7579157 & 2 & 228153974 & C & 0.39 & 1.00 & 0.24 & 0.05 & $9.4 \mathrm{E}-06$ & AGFG1 \\
\hline rs7595130 & 2 & 228151796 & A & 0.39 & 1.00 & 0.24 & 0.05 & $9.5 \mathrm{E}-06$ & AGFG1 \\
\hline rs11674314 & 2 & 228150452 & C & 0.39 & 1.00 & 0.24 & 0.05 & $9.6 \mathrm{E}-06$ & AGFG1 \\
\hline rs4129709 & 2 & 228146793 & A & 0.61 & 1.00 & -0.24 & 0.05 & 9.7E-06 & AGFG1 \\
\hline rs4485561 & 2 & 228081927 & G & 0.43 & 1.00 & 0.24 & 0.05 & $9.8 \mathrm{E}-06$ & AGFG1 \\
\hline
\end{tabular}




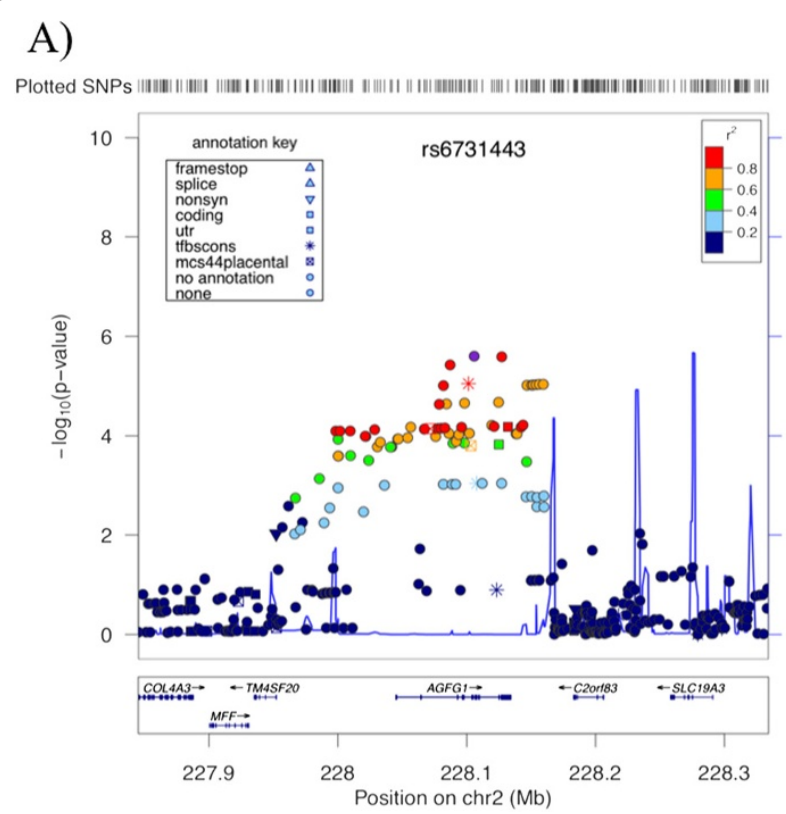

B)

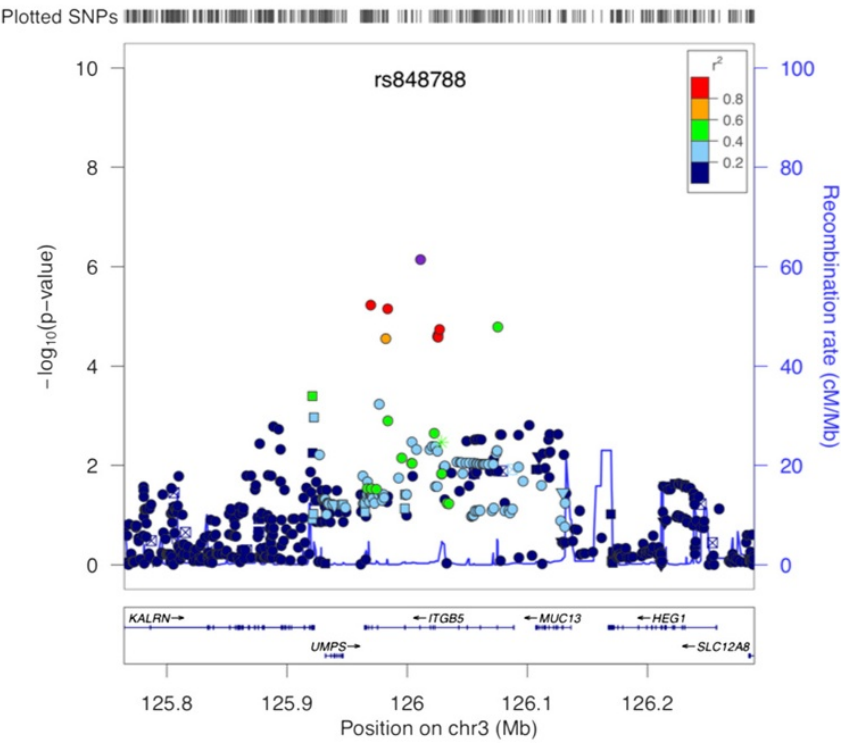

Figure 2 Association regions of SNPs in/near (A) AGFG1 and (B) ITGB5. The x-axes denote position along corresponding chromosomes. The $y$-axes denote - $\log _{10}(P)$ corresponding to primary GWAS P-values of association with AHR. LD between the SNPs with the lowest P-values (rs6731443 and rs848788, in panels (A) and (B), respectively) to each SNP in the plots is denoted by colors and was computed according to hg18/HapMap Phase II CEU data. Plots were created using LocusZoom [21].

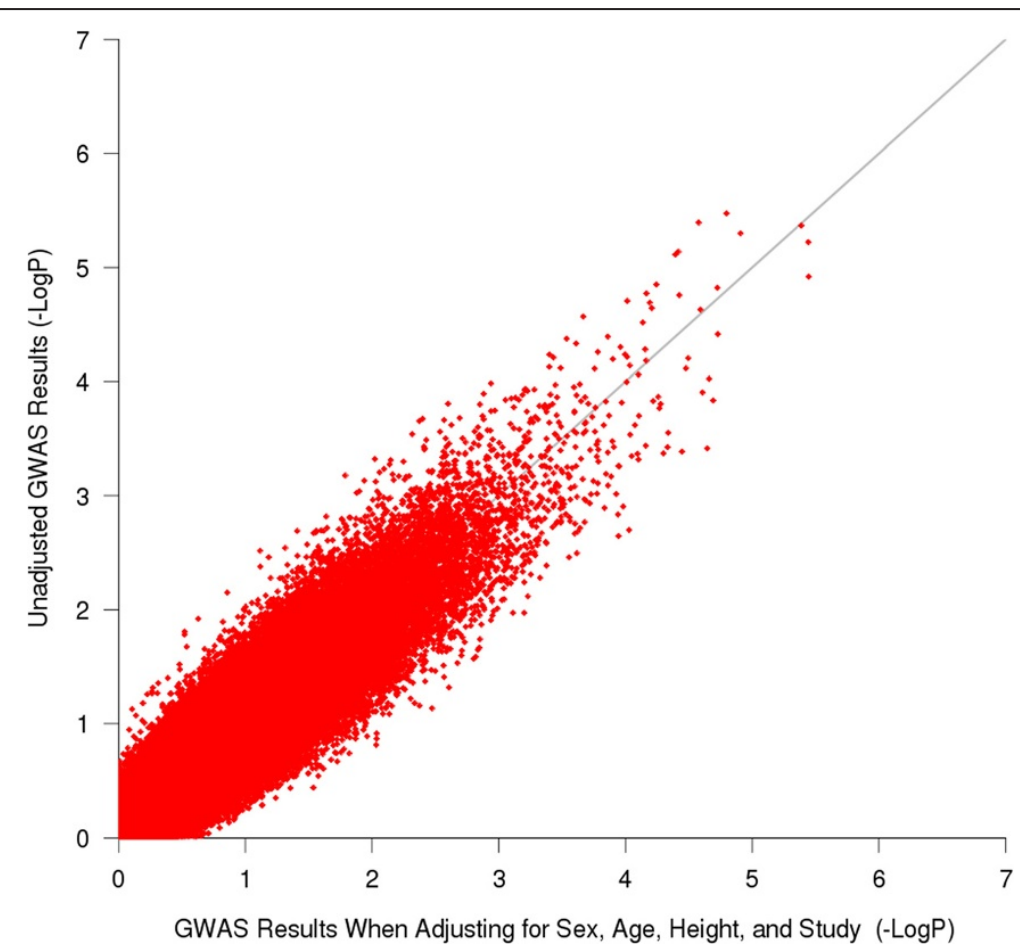

Figure 3 Comparison of P-values generated for genotyped SNPs from the unadjusted primary GWAS vs. those from a model in which sex, age, height, and study were used as covariates. The results reveal that adjustment for these covariates did not drastically alter the ranking or P-values of SNPS. 
Table 3 Attempted replication of top results in independent populations

\begin{tabular}{|c|c|c|c|c|c|c|c|}
\hline \multirow[b]{2}{*}{ SNP } & \multirow{2}{*}{$\begin{array}{l}\text { SHARP } \\
\text { P-value }\end{array}$} & \multicolumn{2}{|c|}{ LHS } & \multicolumn{2}{|c|}{ DAG } & \multirow[b]{2}{*}{ Fisher combined P-value } & \multirow[b]{2}{*}{ AGFG1 eQTL P-value in CAMP } \\
\hline & & Beta & P-value & Beta & P-value & & \\
\hline rs6731443 & $2.5 \mathrm{E}-06$ & -0.022 & 0.012 & -0.026 & 0.39 & $2.14 \mathrm{E}-06$ & 0.0067 \\
\hline rs13382948 & $2.6 \mathrm{E}-06$ & 0.021 & 0.015 & 0.026 & 0.39 & $2.75 \mathrm{E}-06$ & 0.0067 \\
\hline rs10189795 & $3.8 \mathrm{E}-06$ & 0.022 & 0.012 & 0.030 & 0.37 & $2.97 \mathrm{E}-06$ & 0.0052 \\
\hline rs4972983 & $9.0 \mathrm{E}-06$ & -0.021 & 0.017 & -0.034 & 0.35 & $8.62 \mathrm{E}-06$ & 0.0052 \\
\hline rs4485561 & $9.8 \mathrm{E}-06$ & 0.020 & 0.022 & 0.034 & 0.35 & $1.15 \mathrm{E}-05$ & 0.0052 \\
\hline rs848788 & $7.2 \mathrm{E}-07$ & -0.009 & 0.20 & 0.027 & 0.61 & $1.30 \mathrm{E}-05$ & - \\
\hline rs11683662 & 9.3E-06 & -0.020 & 0.025 & 0.043 & 0.68 & $2.21 \mathrm{E}-05$ & - \\
\hline rs7579157 & $9.4 \mathrm{E}-06$ & 0.019 & 0.025 & -0.041 & 0.67 & 2.23E-05 & - \\
\hline rs7595130 & $9.5 \mathrm{E}-06$ & 0.019 & 0.025 & -0.040 & 0.67 & $2.24 \mathrm{E}-05$ & - \\
\hline rs7592613 & $9.3 \mathrm{E}-06$ & -0.020 & 0.026 & 0.042 & 0.68 & $2.25 \mathrm{E}-05$ & - \\
\hline rs4129709 & 9.7E-06 & -0.019 & 0.025 & 0.045 & 0.69 & $2.27 \mathrm{E}-05$ & 0.034 \\
\hline rs11674314 & $9.6 \mathrm{E}-06$ & 0.019 & 0.025 & -0.041 & 0.68 & $2.27 \mathrm{E}-05$ & 0.034 \\
\hline rs614664 & $6.0 \mathrm{E}-06$ & -0.011 & 0.14 & 0.041 & 0.67 & $6.73 \mathrm{E}-05$ & - \\
\hline rs702036 & 7.1E-06 & 0.011 & 0.14 & -0.037 & 0.65 & $7.58 \mathrm{E}-05$ & - \\
\hline rs4861175 & 4.9E-06 & 0.003 & 0.60 & 0.077 & 0.92 & $2.60 \mathrm{E}-04$ & - \\
\hline rs296282 & $8.0 \mathrm{E}-06$ & 0.001 & 0.46 & - & - & - & - \\
\hline
\end{tabular}

Reference alleles are those listed in Table 2. For eQTL, the gene for which transcripts are differentially expressed based on the corresponding SNP is AGFG1. SNPs with no listed eQTL P-values do not meet nominal significance (i.e. P-value $>0.05$ ) for any gene.

To find further support that the associations measured represent biologically relevant findings, we searched for evidence that any are eQTL for the genes in/near them among CAMP subjects who were part of the Asthma BRIDGE genomics study. The SNPs in/near AGFG1 had nominally significant unadjusted P-values for being associated with expression levels of AGFG1 in whole blood [Table 3, Figure 4]. None of these P-values passed Benjamini-Hochberg correction criteria as part of a genome-wide search for eQTL, nor were the expression

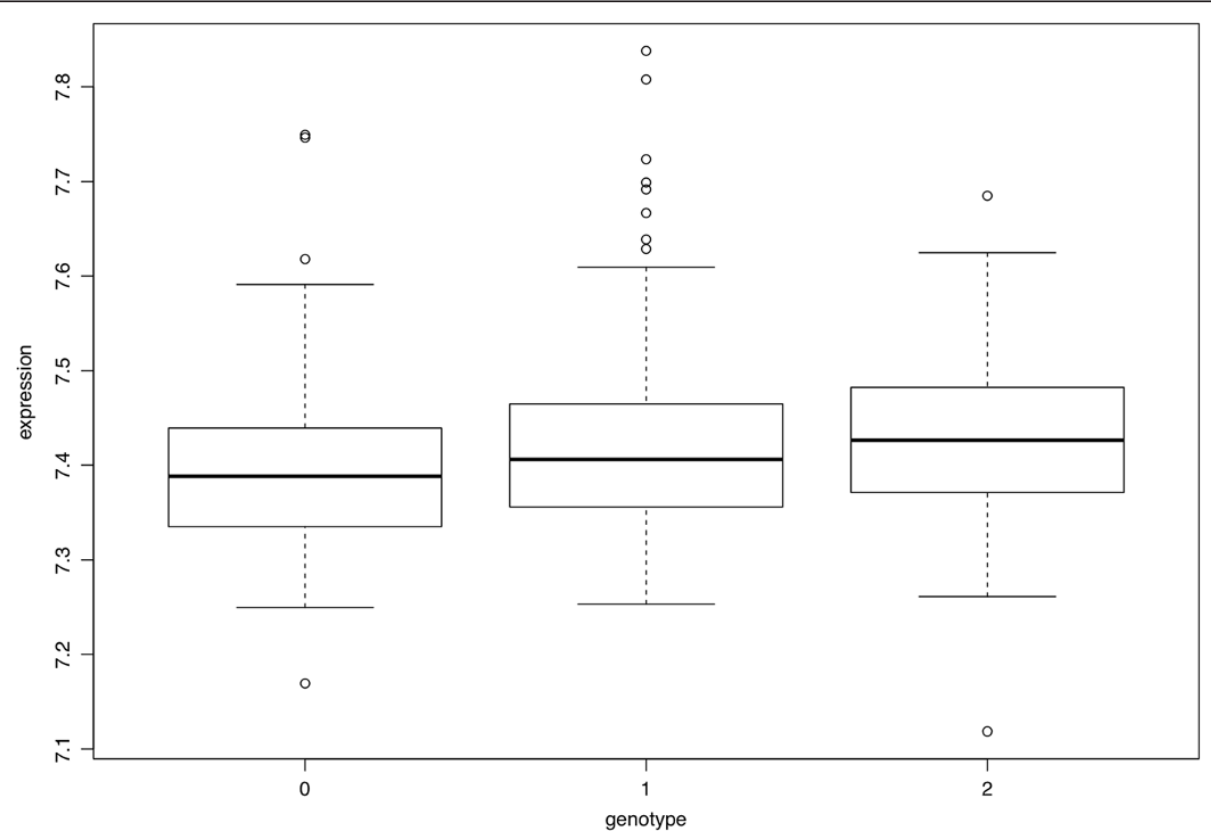

Figure 4 Expression Quantitative Trait Loci (eQTL) results for AGFG1 expression levels by genotype of SNP rs6731443. The genotypes represented along the $x$-axis are GG (0), GT (1) and TT (2). The AGFG1 probe used to plot expression levels has Illumina Probe ID Hoirhennr8UexQTd6l. Plots of AGFG1 eQTLs for other top SNPs in Table 2 are similar to those in this Figure. 
levels of AGFG1 significantly correlated with AHR. None of the other SNPs with P-value $<1 \mathrm{E}-05$ in the primary GWAS had nominally significant eQTL.

\section{Discussion}

Our understanding of asthma genetics has increased in recent years, partly because of the success of large-scale multi-center GWAS [8,9]. Asthma GWAS have identified a robust set of genes that are consistently associated with the disease in diverse populations (e.g. the IKZF3-ZPBP2GSDMB-ORMDL3 locus, TSLP, and RORA), as well as genes that seem to be race/ethnicity specific (e.g. PYHIN1). In addition to studying asthma directly, GWAS have been carried out for related traits, including bronchodilator response [27], plasma IgE concentration [28], and response to inhaled corticosteroids [29]. Compared to asthma GWAS, the study of asthma-related traits has been challenged by the smaller sized cohorts available and the lack of uniform measures of interest across cohorts. While asthma-related trait GWAS often suffer from decreased statistical power due to their smaller sample sizes, they have some advantages over the study of asthma. Asthmarelated traits can be expressed quantitatively, and this may result in increased statistical power to detect associations by capturing phenotypes that do not require arbitrary classification thresholds [30]. Moreover, the investigation of specific intermediate phenotypes of asthma may reduce phenotypic heterogeneity and facilitate the functional validation of association results as some of these traits are more easily simulated in vitro and in animal models [27].

Because AHR is a complex phenotype, our GWAS results are limited by our ability to properly define it in subjects. In a previous study of CAMP subjects, the repeatability of PC20 measures over the 4-year length of the clinical trial was high, as demonstrated by intraclass correlation coefficients, after adjusting for age, race/ethnicity, height, family income, parental education, and symptom score, of 0.67 for subjects taking budesonide or placebo and 0.73 for subjects taking nedocromil [31]. A twin study of methacholine responsiveness found that the intrapair correlation coefficient among monozygotic twins (0.67) was significantly greater than that for dizygotic twins (0.34) [12]. Positional cloning and linkage analysis studies of AHR have identified two well-validated asthma candidate genes: ADAM33 [13] and PCDH1 [14]. Although the high intraclass correlation of AHR measures and previous twin and association studies suggest that AHR is a heritable trait, AHR varies in time within individuals and is influenced by environmental factors and medication usage. Among the baseline AHR measures used in our GWAS, some were taken after placebo washout periods of varying length, while others were taken in subjects (e.g. some from CARE) who were not necessarily off of asthma medications [Table 1]. This difference would decrease our ability to detect significant relationships, such that less significant associations would be detected than if all subjects had had a placebo washout.

We performed our GWAS using subjects with AHR measures from as many asthma trials as possible in order to maximize our statistical power to detect associations. Nonetheless, partly because AHR is not always measured in asthma clinical trials, our sample size was limited to 994. One significant difference among the trials we utilized is that the CAMP inclusion criteria included having AHR, such that $12.5 \mathrm{mg} / \mathrm{dl}$ of methacholine or less resulted in a $20 \%$ drop in $\mathrm{FEV}_{1}$. Nonetheless, CAMP and CARE had similarly severe AHR (mean LnPC20 0.096 (SD 1.21) and -0.25 (SD 1.35), respectively). CAMP and CARE were also similar in terms of age, sex, and baseline $\mathrm{FEV}_{1}$ [Table 1]. These two pediatric populations contrast with ACRN, which was composed mostly of adults, had a greater proportion of female participants, less severe AHR (mean LnPC20 0.80 (SD 0.68)), and lower baseline $\mathrm{FEV}_{1}$. We investigated some of the effects of trial heterogeneity on our primary association results by performing the GWAS while adjusting for sex, age, height, and study. As Figure 3 shows, these covariates did not have a strong influence on the ranks or P-values obtained. Because performing the adjustment for covariates limited our sample size further to 989 due to missing demographic variable entries for some subjects, we opted to utilize the unadjusted results.

The replication populations were also distinct from our primary populations. DAG quantified AHR differently than all other cohorts, as the slope defined by the change in $\mathrm{FEV}_{1}$ between the stimulant dose step at which a $20 \%$ or greater $\mathrm{FEV}_{1}$ drop was achieved vs. baseline, divided by the dose of stimulant used. This definition allowed for the inclusion of subjects who did not achieve a PC20 at the doses of stimulants administered, and hence, allowed for the inclusion of subjects with less severe AHR. Fifty of 650 DAG subjects did not have PC20 measures, and hence, the estimate of mean LnPC20 provided in Table 1 is biased in that it does not include the measures of patients with less severe AHR. The AHR severity among LHS participants was also lower, on average, than that for primary cohort subjects (mean LnPC20 1.58 (SD 0.93)). While no participants of CAMP, CARE, or ACRN were smokers at the time of baseline AHR measures, LHS was a clinical trial that specifically enrolled adult smokers with COPD, while DAG was an observational study that included both smokers and non-smokers with asthma. Because smoking is known to affect lung function, AHR measures in both of these cohorts are likely affected by the smoking status of their participants. Further, LHS was not composed of asthma patients, and hence, it is likely that some of the 
biological mechanisms underlying its subjects AHR do not overlap with those of asthma patients. Despite the large differences among cohorts, we attempted to replicate our primary findings in LHS and DAG to assess their generalizability.

For the primary GWAS, four independent regions had P-values $<1 \mathrm{E}-05$ [Table 2]. While due to the small sample size of our primary GWAS, it is not surprising that none of these regions met traditional genome-wide significance levels, two of the top regions were composed of multiple SNPs within two genes (i.e. AGFG1 and ITGB5), which increased the likelihood that the associations were biologically significant. The top ITGB5 association was at SNP rs848788 (P-value 7.2E-07), while the top AGFG1 association was at SNP rs6731443 (P-value 2.5E-06), both intronic SNPs of the corresponding genes. We utilized the AceView tool [32] to gather current information for these genes. The ITGB5 gene maps to chromosome 3 at 3q21.2, and covers $139.46 \mathrm{~kb}$, from 124620254 to 124480791 (NCBI 37, August 2010). ITGB5 is a highly expressed gene in many tissues, including lung, with 15 different mRNAs and 10 probable alternative promoters. The gene has been studied widely and its product, integrin $\beta 5$, is involved in cell adhesion and integrin-mediated signaling. Of most relevance to AHR, one study by Tatler, et al., investigated whether contraction agonists could promote TGF- $\beta$ activation in human airway smooth muscle (HASM) cells and found that integrin $\alpha \nu \beta 5$ was a mediator of this activation [33]. Specifically, lysophosphatidic acid (LPA) and methacholine activation of TGF- $\beta$, a cytokine that has been implicated in airway remodeling in asthma, was shown to be abrogated by an $\alpha v \beta 5$ blocking antibody. Further, the $\beta 5$ cytoplasmic domain of integrin $\alpha v \beta 5$ was found to be involved in LPA activation because a polymorphism in this subunit reversed the integrin $\alpha v \beta 5$ activation of TGF- $\beta$. Comparison of normal and asthmatic HASM cells found that asthmatic HASM had increased LPA-induced, integrin $\alpha v \beta 5$-mediated TGF- $\beta$ activity, and that this increase was not due to increased cell surface expression of integrin $\alpha v \beta 5$. While we were unable to replicate the primary GWAS ITGB5 associations in two independent populations, and there was no evidence that the corresponding SNPs were eQTL in whole blood of CAMP subjects, the study by Tatler, et al., suggests that polymorphisms in ITGB5 could play a role in modulating HASM response to contraction agonists via mechanisms that do not involve changes to the expression levels of the genes whose products form integrin $\alpha v \beta 5$. Further, it is possible that our top ITGB5 SNPs are eQTLs of this gene HASM cells and not in whole blood, which is the tissue for which we currently have eQTL measures. Thus, it is possible that the ITGBS association we observed truly reflects a genetic change that modulates AHR.
Our association results for AGFG1 variants were stronger than for ITGB5 ones in that we were able to replicate them in one of two independent populations (lowest combined P-value across all cohorts was rs6731443 2.14E-06) and the corresponding SNPs were nominally significant eQTL of AGFG1. The AGFG1 gene maps to chromosome 2 , at $2 \mathrm{q} 36.3$, covering $89.07 \mathrm{~kb}$, from 228336873 to 228425938 (NCBI 37, August 2010). AGFG1 is also a highly expressed gene in many tissues, including lung, with 17 different mRNAs and 4 probable alternative promoters. The protein encoded by this gene (a.k.a. RIP, $H R B$ ) binds the activation domain of the human immunodeficiency virus (HIV) Rev protein when Rev is assembled onto its RNA target, and is required for the nuclear export of Revdirected RNAs [34]. While many studies of AGFG1 focus on its relationship to HIV, AGFG1 has also been found to be involved in influenza A genome trafficking from the host nucleus to plasma membrane [35]. Because asthma development and severity are known to be influenced by respiratory pathogens, including influenza [36], current knowledge of AGFG1 suggests that if our association and eQTL data for this gene represent true biologically significant findings, their relationship with AHR may involve changes in immune response or susceptibility to external factors (e.g., influenza). Further functional validation of the eQTL results, including via quantitative RT-PCR of AGFG1 in subjects with various genotypes of the SNPs listed in Table 3 and for various tissues/cell types, would help clarify whether any relationship between the identified AGFG1 variants and its expression levels truly exists. Because the nominal replication of AGFG1 associations was observed in LHS, a clinical trial that measured baseline AHR in smokers who did not have current asthma, it is possible that the AGFG1 association reflects biological processes modulating AHR that are not unique to asthma.

\section{Conclusions}

An AHR GWAS among 994 asthma patients found that the most strongly associated SNPs, rs848788 (P-value 7.2E-07) and rs6731443 (P-value 2.5E-06), were in the ITGB5 and AGFG1 genes, respectively. The ITGB5 association did not replicate in two independent populations, nor was there any evidence that the corresponding SNP was an eQTL of ITGB5. The AGFG1 result replicated at a nominally significant level in one independent population of COPD subjects (LHS P-value 0.012), and the SNP had a nominally significant unadjusted P-value (0.0067) for being eQTL of AGFG1. While future functional studies are required to validate the potential involvement of these SNPs in modulating AHR, current knowledge of both genes suggests that our associations may represent biologically significant findings. 


\section{Additional file}

\section{Additional file 1: Online supplement.}

\begin{abstract}
Abbreviations
ACRN: Asthma Clinical Research Network; ADAM33: ADAM metallopeptidase domain 33; AGFG1: ArfGAP with FG repeats 1; AHR: Airway hyperresponsiveness; CAMP: Childhood Asthma Management Program; CARE: Childhood Asthma Research and Education; DAG: Dutch Asthma GWAS; eQTL: Expression quantitative trait loci; FEV ${ }_{1}$ : Forced expiratory volume in one second; FVC: Forced vital capacity; GWAS: Genome-wide association study; GSDMB: Gasdermin B; HASM: Human airway smooth muscle; HLA-DQ: Major histocompatibility complex, class II, DQ; IKZF3: IKAROS family zinc finger 3 (Aiolos); IL 1RL 1: Interleukin 1 receptor-like 1; IL33: Interleukin 33; ITGB5: Integrin, beta 5; LHS: Lung Health Study; LnPC20: Natural log of methacholine concentration causing a 20\% decrease in $\mathrm{FEV}_{1}$; LPA: Lysophosphatidic acid; MAF: Minor allele frequency; ORMDL3: ORM1-like 3 (S. cerevisiae); PCDH1: Protocadherin 1; PYHIN1: Pyrin and HIN domain family, member 1; QC: Quality control; QQ: Quantilequantile; RORA: RAR-related orphan receptor A; SHARP: NHLBI SNP Health Association Resource Asthma Resource project; SLC22A5: Solute carrier family 22 (organic cation/carnitine transporter), member 5; SMAD3: SMAD family member 3; SNPS: Single nucleotide polymorphisms; TSLP: Thymic stromal lymphopoietin; ZPBP2: Zona pellucida binding protein 2.
\end{abstract}

\section{Competing interests}

The authors declare that they have no competing interests.

\section{Authors' contributions}

$\mathrm{BEH}$ designed the study, analyzed primary data, and drafted the manuscript. $B R, K G T$, and STW contributed to the overall study concept and design. WQ provided statistical support. BK, JZ, and JS processed genetic data of primary populations. SJS, RFL, RSZ, RCS, FDM, HB, VMC, El, and DM collected subjects for and designed primary cohort clinical trials. GHK, MAEN, DSP and JMV conducted the association study in DAG. NR, NNH, KB conducted the association study in LHS. All authors edited the manuscript and approved its final draft.

\section{Acknowledgements}

We thank all CAMP subjects for their ongoing participation in this study. We acknowledge the CAMP investigators and research team, supported by the National Heart, Lung and Blood Institute (NHLBI), for collection of CAMP Genetic Ancillary Study data. All work on data collected from the CAMP Genetic Ancillary Study was conducted at the Channing Laboratory of the Brigham and Women's Hospital under appropriate CAMP policies and human subject's protections. The CAMP Genetics Ancillary Study is supported by U01 HL075419, U01 HL65899, P01 HL083069, R01 HL086601, and T32 HL07427 from the NHLBI, National Institutes of Health (NIH). Additional support was provided by NIH U10 HL064287, U10 HL064288, U10 HL064295, U10 HL064305, U10 HL064307, U01 HL064313, RC2 HL101487, and U01 HL65899, an NIH Pharmacogenomics Research Network (PGRN) - RIKEN Center for Genomic Medicine (CGM) Global Alliance. BEH was supported by $\mathrm{NIH}$ K99 HL105663. DAG was supported by the Netherlands Asthma Foundation grant AF (AF 95.09, AF 98.48, AF 3.2.02.51 and AF 3.2.07.015) and a grant from the University Medical Center Groningen. GHK was supported by a Ter Meulen Fund grant from the Royal Netherlands Academy of Arts and Sciences. The Lung Health Study I was supported by contract NIH/N01HR-46002 and genotyping of its subjects was supported by GENEVA (U01HG004738). KCB was supported in part by the Mary Beryl Patch Turnbull Scholar Program.

\section{Author details}

'Channing Division of Network Medicine, Brigham and Women's Hospital and Harvard Medical School, Boston, MA, USA. ${ }^{2}$ Partners HealthCare Center for Personalized Genetic Medicine and Harvard Medical School, Boston, MA, USA. ${ }^{3}$ Children's Hospital Informatics Program, Boston, MA, USA. ${ }^{4}$ National Jewish Health and University of Colorado Denver School of Medicine, Denver, CO, USA. ${ }^{5}$ University of Wisconsin, Clinical Science Center, Madison, WI, USA. ${ }^{6}$ Kaiser Permanente Southern California Region, San Diego, CA, USA. ${ }^{7}$ Washington University School of Medicine, St. Louis, MO, USA. ${ }^{8}$ Arizona
Respiratory Center, University of Arizona, College of Medicine, Tucson, AZ, USA. ${ }^{9}$ Division of Pulmonary/Critical Care and Allergy/Immunology, Department of Medicine, University of California at San Francisco, San Francisco, CA, USA. ${ }^{10}$ Department of Biostatistics, Penn State College of Medicine, Hershey, PA, USA. ${ }^{11}$ Division of Pulmonary and Critical Care Medicine, Department of Medicine, Brigham and Women's Hospital, Boston, MA, USA. ${ }^{12}$ Department of Pediatric Pulmonology and Pediatric Allergology, Beatrix Children's Hospital, GRIAC Research Institute, University of Groningen, University Medical Center Groningen, Groningen, the Netherlands. ${ }^{13}$ Department of Pulmonology and Tuberculosis, GRIAC Research Institute, University of Groningen, University Medical Center Groningen, Groningen, the Netherlands. ${ }^{14}$ Department of Epidemiology, GRIAC Research Institute, University of Groningen, University Medical Center Groningen, Groningen, the Netherlands. ${ }^{15}$ Department of Medicine, Johns Hopkins University, Baltimore, MD, USA.

Received: 29 August 2012 Accepted: 22 August 2013 Published: 28 August 2013

\section{References}

1. Akinbami L, Moorman J, Bailey C, Zahran H, King M, Johnson C, Liu X: Book Trends in asthma prevalence, health care use, and mortality in the United States, 2001-2010. Baltimore, MD: National Center for Health Statistics data brief, no 94; 2012.

2. Hargreave FE, Ryan G, Thomson NC, O'Byrne PM, Latimer K, Juniper EF, Dolovich J: Bronchial responsiveness to histamine or methacholine in asthma: measurement and clinical significance. J Allergy Clin Immunol 1981, 68:347-355.

3. Wu AC, Tantisira K, Li L, Schuemann B, Weiss ST, Fuhlbrigge AL: Predictors of symptoms are different from predictors of severe exacerbations from asthma in children. Chest 2011, 140:100-107.

4. Tantisira KG, Fuhlbrigge AL, Tonascia J, Van Natta M, Zeiger RS, Strunk RC, Szefler SJ, Weiss ST: Bronchodilation and bronchoconstriction: predictors of future lung function in childhood asthma. J Allergy Clin Immunol 2006, 117:1264-1271.

5. Cockcroft DW: Direct challenge tests: Airway hyperresponsiveness in asthma: its measurement and clinical significance. Chest 2010, 138:18S-24S.

6. Cockcroft DW, Davis BE: Mechanisms of airway hyperresponsiveness. J Allergy Clin Immunol 2006, 118:551-559. quiz 560-551.

7. Busse WW: The relationship of airway hyperresponsiveness and airway inflammation: Airway hyperresponsiveness in asthma: its measurement and clinical significance. Chest 2010, 138:4S-10S.

8. Moffatt MF, Gut IG, Demenais F, Strachan DP, Bouzigon E, Heath S, von Mutius E, Farrall M, Lathrop M, Cookson WO: A large-scale, consortiumbased genomewide association study of asthma. N Engl J Med 2010, 363:1211-1221.

9. Torgerson DG, Ampleford EJ, Chiu GY, Gauderman WJ, Gignoux CR, Graves PE, Himes BE, Levin AM, Mathias RA, Hancock DB, et al: Meta-analysis of genome-wide association studies of asthma in ethnically diverse North American populations. Nat Genet 2011, 43:887-892.

10. Zosky GR, Larcombe AN, White OJ, Burchell JT, Janosi TZ, Hantos Z, Holt PG, Sly PD, Turner DJ: Ovalbumin-sensitized mice are good models for airway hyperresponsiveness but not acute physiological responses to allergen inhalation. Clin Exp Allergy 2008, 38:829-838.

11. Wills-Karp M, Ewart SL: The genetics of allergen-induced airway hyperresponsiveness in mice. Am J Respir Crit Care Med 1997, 156:S89-S96.

12. Hopp RJ, Bewtra AK, Watt GD, Nair NM, Townley RG: Genetic analysis of allergic disease in twins. J Allergy Clin Immunol 1984, 73:265-270.

13. Van Eerdewegh P, Little RD, Dupuis J, Del Mastro RG, Falls K, Simon J, Torrey $D$, Pandit S, McKenny J, Braunschweiger K, et al: Association of the ADAM33 gene with asthma and bronchial hyperresponsiveness. Nature 2002, 418:426-430.

14. Koppelman GH, Meyers DA, Howard TD, Zheng SL, Hawkins GA, Ampleford EJ, Xu J, Koning H, Bruinenberg M, Nolte IM, et al: Identification of PCDH1 as a novel susceptibility gene for bronchial hyperresponsiveness. Am J Respir Crit Care Med 2009, 180:929-935.

15. The Childhood Asthma Management Program (CAMP): design, rationale, and methods: Childhood Asthma Management Program Research Group. Control Clin Trials 1999, 20:91-120. 
16. Guilbert TW, Morgan WJ, Krawiec M, Lemanske RF Jr, Sorkness C, Szefler SJ, Larsen G, Spahn JD, Zeiger RS, Heldt G, et al: The prevention of early asthma in kids study: design, rationale and methods for the childhood asthma research and education network. Control Clin Trials 2004, 25:286-310.

17. Szefler SJ, Chinchilli VM, Israel E, Denlinger LC, Lemanske RF Jr, Calhoun W, Peters SP: Key observations from the NHLBI asthma clinical research network. Thorax 2012, 67:450-455.

18. American Thoracic Society: Lung function testing: selection of reference values and interpretative strategies. Am Rev Respir Dis 1991, 144:1202-1218.

19. Willer CJ, Sanna S, Jackson AU, Scuteri A, Bonnycastle LL, Clarke R, Heath SC, Timpson NJ, Najjar SS, Stringham HM, et al: Newly identified loci that influence lipid concentrations and risk of coronary artery disease. Nat Genet 2008, 40:161-169.

20. Purcell S, Neale B, Todd-Brown K, Thomas L, Ferreira MA, Bender D, Maller J, Sklar P, de Bakker PI, Daly MJ, Sham PC: PLINK: a tool set for wholegenome association and population-based linkage analyses. Am J Hum Genet 2007, 81:559-575.

21. Pruim RJ, Welch RP, Sanna S, Teslovich TM, Chines PS, Gliedt TP, Boehnke M, Abecasis GR, Willer CJ: LocusZoom: regional visualization of genome-wide association scan results. Bioinformatics 2010, 26:2336-2337.

22. Fisher RA: Combining independent tests of significance. Am Statistician 1948, 2:30.

23. R Development Core Team: R: A Language and Environment for Statistical Computing. Vienna, Austria: R Foundation for Statistical Computing; 2008.

24. Vonk JM, Postma DS, Maarsingh $\mathrm{H}$, Bruinenberg $\mathrm{M}$, Koppelman GH, Meurs $\mathrm{H}$ : Arginase 1 and arginase 2 variations associate with asthma, asthma severity and beta2 agonist and steroid response. Pharmacogenet Genomics 2010, 20:179-186.

25. Connett JE, Kusek JW, Bailey WC, O'Hara P, Wu M: Design of the lung health study: a randomized clinical trial of early intervention for chronic obstructive pulmonary disease. Control Clin Trials 1993, 14:3S-19S.

26. Tashkin DP, Altose MD, Bleecker ER, Connett JE, Kanner RE, Lee WW, Wise R: The lung health study: airway responsiveness to inhaled methacholine in smokers with mild to moderate airflow limitation. The Lung Health Study Research Group. Am Rev Respir Dis 1992, 145:301-310.

27. Himes BE, Jiang X, Hu R, Wu AC, Lasky-Su J, Klanderman B, Ziniti J, SenterSylvia J, Lima J, Irvin CG, Weiss ST: Genome-wide association analysis in asthma subjects identifies SPATS2L as a novel bronchodilator response gene. PLoS Genet 2012, 8(7):e1002824.

28. Granada M, Wilk JB, Tuzova M, Strachan DP, Weidinger S, Albrecht E, Gieger C, Heinrich J, Himes BE, Hunninghake GM, et al: A genome-wide association study of plasma total IgE concentrations in the Framingham Heart Study. J Allergy Clin Immunol 2011, 129:840-845. e821.

29. Tantisira KG, Lasky-Su J, Harada M, Murphy A, Litonjua AA, Himes BE, Lange C, Lazarus R, Sylvia J, Klanderman B, et al: Genomewide association between GLCCI1 and response to glucocorticoid therapy in asthma. N Engl J Med 2011, 365:1173-1183

30. Blangero J: Genetic analysis of a common oligogenic trait with quantitative correlates: summary of GAW9 results. Genet Epidemiol 1995, 12:689-706.

31. Wu AC, Tantisira K, Li L, Schuemann B, Weiss S: Repeatability of response to asthma medications. J Allergy Clin Immunol 2009, 123:385-390.

32. Thierry-Mieg D, Thierry-Mieg J: AceView: a comprehensive cDNA-supported gene and transcripts annotation. Genome Biol 2006, 7(Suppl 1):S12 11-14.

33. Tatler AL, John AE, Jolly L, Habgood A, Porte J, Brightling C, Knox AJ, Pang L, Sheppard D, Huang X, Jenkins G: Integrin alphavbeta5-mediated TGF-beta activation by airway smooth muscle cells in asthma. J Immunol 2011, 187:6094-6107.

34. Sanchez-Velar N, Udofia EB, Yu Z, Zapp ML: hRIP, a cellular cofactor for Rev function, promotes release of HIV RNAs from the perinuclear region. Genes Dev 2004, 18:23-34.

35. Eisfeld AJ, Neumann G, Kawaoka Y: Human immunodeficiency virus revbinding protein is essential for influenza a virus replication and promotes genome trafficking in late-stage infection. J Virol 2011, 85:9588-9598.

36. Edwards MR, Bartlett NW, Hussell T, Openshaw P, Johnston SL: The microbiology of asthma. Nat Rev Microbiol 2012, 10(7):459-471.

\section{Submit your next manuscript to BioMed Central and take full advantage of:}

- Convenient online submission

- Thorough peer review

- No space constraints or color figure charges

- Immediate publication on acceptance

- Inclusion in PubMed, CAS, Scopus and Google Scholar

- Research which is freely available for redistribution 